\title{
El fisioterapeuta en la Unidad de Cuidados Intensivos ¿un profesional necesario?
}

\author{
Is the physiotherapist in the Intensive Care Unit a necessary professional?
}

\author{
Miguel Ángel Martínez Camacho, * Robert Alexander Jones Baro, * \\ Alberto Gómez González*
}

El paciente ingresado en la Unidad de Cuidados Intensivos (UCI) desarrolla, en la mayoría de los casos, una serie de complicaciones derivadas de la inmovilidad, la ventilación mecánica, los fármacos utilizados, malnutrición, procesos inflamatorios sistémicos, entre muchas otras cosas; esto afecta la funcionalidad, la calidad de vida y el grado de dependencia, pudiendo culminar en una discapacidad permanente. ${ }^{1}$ Los pacientes ingresados en la $\mathrm{UCl}$ presentan un proceso de desacondicionamiento físico, pérdida de fuerza y masa muscular, reducción en los volúmenes pulmonares, reducción en la motilidad intestina y una gran lista de afectaciones multisistémicas, además de estar en alto riesgo de desarrollar escaras por presión, neumonías asociadas con la ventilación mecánica, alteraciones musculoesqueléticas derivadas de un mal posicionamiento, etcétera. Una entidad clínica comúnmente observada en el paciente crítico es la debilidad adquirida en la $\mathrm{UCl}$, la cual se puede generar por una afectación muscular, nerviosa o mixta (polineuropatía del paciente crítico y/o miopatía del paciente crítico), manifestándose en la musculatura estriada, desde las extremidades hasta los músculos respiratorios, y generando un aumento de días de estancia en $\mathrm{UCI}$, días de ventilación mecánica, costos y alteraciones funcionales al alta hospitalaria. ${ }^{1,2}$

Si bien el manejo del paciente en estado crítico es responsabilidad de un equipo multidisciplinario especializado que abarca desde el médico intensivista, médico fisiatra, enfermeros, nutriólogos, etcétera, el fisioterapeuta es una pieza clave en la reducción de las secuelas generadas por el internamiento en la $\cup \mathrm{Cl}$. Existen tres ejes centrales generales en el manejo fisioterapéutico de los pacientes críticos, éstos son: la movilización

\footnotetext{
* Licenciatura en Fisioterapia, Universidad Autónoma de Querétaro, México.
}

Correspondencia:

Miguel Ángel Martínez Camacho

Correo electrónico: Iftmiguelangelmtz@gmail.com

Aceptado: 13-01-2020.

www.medigraphic.com/actamedica

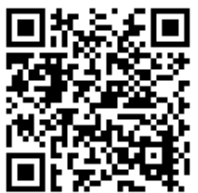

temprana, el entrenamiento de músculos inspiratorios y las técnicas de higiene bronquial (fisioterapia de tórax).

De manera clásica, se ha asociado exclusivamente al fisioterapeuta en la UCI con técnicas de higiene bronquial, lo cual forma parte de sus competencias, aunque limitarlo sólo en esto es desperdiciar un recurso valioso. En un reciente metaanálisis no se observó una reducción en las neumonías asociadas con la ventilación mecánica mediante técnicas de fisioterapia de tórax, sin embargo, sí hubo una reducción en la mortalidad. 3,4 Según la Asociación Americana de Cuidados Respiratorios, no se recomienda el uso rutinario de técnicas de higiene bronquial en todos los pacientes hospitalizados, sino que su uso dependerá de la condición particular de cada persona, como es el caso de pacientes con hipersecreción bronquial; en éstos, las técnicas de higiene bronquial se pudieran recomendar, o también en patología neuromuscular, en la que el asistente de tos (cough assist) está indicado. ${ }^{5}$ Otro dispositivo ampliamente difundido para la prevención de complicaciones durante la hospitalización, sobre todo en pacientes postquirúrgicos, es el inspirómetro de incentivo, el cual no tiene una evidencia sólida para su implementación general., 5,

El entrenamiento de los músculos inspiratorios consiste en la administración de una carga extra a dicha musculatura, tomando como referencia un tanto por ciento de la presión inspiratoria máxima (generalmente entre el 30 al 50\%). Este tipo de entrenamiento puede ser de gran ayuda para el retiro de la ventilación mecánica, sobre todo en aquéllos con destete difícil.7,8 El entrenamiento de los músculos inspiratorios se realiza habitualmente por medio de un dispositivo llamada Threshold, el cual se acopla al tubo endotraqueal o a la cánula de traqueostomía, en el caso de pacientes bajo ventilación mecánica, o directamente mediante una boquilla y una pinza nasal en los pacientes sin ventilación mecánica.

La movilización temprana es una estrategia segura, ${ }^{1,9}$ ampliamente difundida y es considerada como un estándar general en el manejo del paciente críticamente enfermo alrededor del mundo. La movilización temprana se define como la aplicación de actividad física dentro de los primeros dos a tres días del inicio de la enfermedad grave o de la lesión. ${ }^{1}$ Cabe mencionar 
que se debe privilegiar la contracción activa por parte del paciente, siempre basándose en el nivel de fuerza muscular y de movilidad. La movilización temprana ayuda en la prevención de la debilidad adquirida en la $\mathrm{UCl}$, promueve la reducción de la sedación, delírium, trombosis venosa profunda, neumonía asociada con la ventilación mecánica, escaras por presión y, en algunos estudios, se ha reportado reducción de la estancia hospitalaria y de la estancia en la $\mathrm{UCl}$, además de aumento de los días libres de ventilador, mejoría en la fuerza muscular $y$ en el grado de independencia al alta hospitalaria. ${ }^{10-12}$ Esta última parte es de suma importancia si reflexionamos que, según el Acuerdo Relativo a los Costos Unitarios por Nivel de Atención Médica actualizado hasta el año 2019 y publicado en el Diario Oficial de la Federación, se determina que el día de hospitalización en un centro de segundo nivel en México tiene un costo de $\$ 8,333$ pesos ( $\$ 434.71$ USD), mientras que un día en terapia intensiva tiene un costo de $\$ 37,410$ pesos mexicanos (\$1951.59 USD). Si a esto se le suma que la movilización favorece la función pulmonar, mejora el manejo de secreciones y aumenta la fuerza de los músculos de las extremidades, tronco y los músculos respiratorios, ${ }^{7}$ queda claro que privar al paciente de este beneficio es un error garrafal, incluso rozando la iatrogenia. Un paciente que se mantiene en movimiento desarrollará un mejor desenlace clínico, además habrá una reducción de gastos y se preverá la discapacidad. 1,10,11

Durante mucho tiempo los esfuerzos del equipo de rehabilitación se han enfocado en cuestiones pasivas como el posicionamiento, la prevención de escaras por presión, uso de tromboprofilaxis mecánica mediante medias de compresión o el uso de dispositivos de presión intermitente y la elaboración de férulas para evitar posiciones inadecuadas en las articulaciones, sin embargo, la tendencia actual en medicina crítica conocida como "menos es más" abre la puerta para un manejo más activo del paciente:12,13 menos sedación, menos catéteres y sondas, menos monitorización invasiva y, en general, hacer cuidados de alto valor basados en las intervenciones que realmente benefician al paciente y desechando aquéllas que no tienen beneficio probado o que incluso ponen al paciente en riesgo de morir o tener alguna secuela funcional permanente. ${ }^{13-15}$ Existe un pequeño grupo de pacientes que tienen indicación para sedación profunda, lo cual limita la cooperación durante la intervención de la fisioterapia y la convierte en medidas preventivas-pasivas como las comentadas anteriormente. En la actualidad estos pacientes deben ser la minoría, sin embargo, en caso de no ser así, sería un buen ejercicio preguntarnos si nuestra $\mathrm{UCl}$ cuenta con buenos protocolos de sedación. ${ }^{15}$

Cotidianamente, el fisioterapeuta es un excelente aliado en problemas que se presentan en la $\mathrm{UCl}$. Al decir esto, se vuelve razonable recalcar la necesidad de un fisioterapeuta con conocimientos en cuidados intensivos para el manejo exclusivo de estos pacientes. El fisioterapeuta es experto en el movimiento y la funcionalidad, permitamos al paciente recibir este beneficio desde la fase aguda de la enfermedad, pues es mejor prevenir secuelas que tratar de remediarlas. El ingreso de un paciente a la UCI le debe brindar la posibilidad de seguir viviendo de manera digna, salvaguardando paralelamente la vida y la funcionalidad.

Una $\mathrm{UCI}$ sin fisioterapeutas es una $\mathrm{UCl}$ incompleta.

\section{REFERENCIAS}

1. Cameron S, Ball I, Cepinskas G, Choong K, Doherty TJ, Ellis Christopher $G$ et al. Early mobilization in the critical care unit: a review of adult and pediatric literatura. J Crit Care. 2015; 30 (4): 664-672. doi: 10.1016/j. jcrc.2015.03.032.

2. Winkelman C. Bed rest in health and critical illness a body system approach. AACN Adv Crit Care. 2009; 20 (3): 254-266. doi: 10.1097/ $\mathrm{NCl} .0 \mathrm{~b} 013 \mathrm{e} 3181 \mathrm{ac} 838 \mathrm{~d}$.

3. Wang MY, Pan L, Hu XJ. Chest physiotherapy for prevention of ventilator-associated pneumonia: a meta-analysis. Am J Infect Control. 2018; 47 (7): 755-760. https://doi.org/10.1016/j.ajic.2018.12.015.

4. Pozuelo-Carrascosa DP, Torres-Costoso A, Alvarez-Bueno C, CaveroRedondo I, López Muñoz P, Martínez-Vizcaíno V. Multimodality respiratory physiotherapy reduces mortality but may not prevent ventilator-associated pneumonia or reduce length of stay in the intensive care unit: a systematic review. J Physiother. 2018; 64 (4): 222-228. doi: 10.1016/j.jphys.2018.08.005.

5. Strickland SL, Rubin BK, Drescher GS, Haas CF, O'Malley CA, Volsko TA et al. AARC clinical practice guideline: effectiveness of nonpharmacologic airway clearance therapies in hospitalized patients. Respir Care. 2013; 58 (12): 2187-2193. doi: 10.4187/respcare.02925.

6. Do Nascimento Junior P, Módolo NS, Andrade S, Guimarães MM, Braz LG, El Dib R. Incentive spirometry for prevention of postoperative pulmonary complications in upper abdominal surgery. Cochrane Database Syst Rev. 2014; (2): CD006058. doi: 10.1002/14651858.CD006058.pub3.

7. Elkins $M$, Dentice R. Inspiratory muscle training facilitates weaning from mechanical ventilation among patients in the intensive care unit: a systematic review. J Physiother. 2015; 61: 125-134. doi: http://dx.doi. org/10.1016/j.jphys.2015.05.016.

8. Vorona S, Sabatini U, Al-Maqbali S, Bertoni M, Dres M, Bissett B et al. Inspiratory muscle rehabilitation in critically ill adults. A systematic review and meta-analysis. Ann Am Thorac Soc. 2018; 15 (6): 735-744. doi:10.1513/AnnalsATS.201712-961OC.

9. Hodgson CL, Stiller K, Needham DM, Tipping CJ, Harrold M, Baldwin $\mathrm{CE}$ et al. Expert consensus and recommendations on safety criteria for active mobilization of mechanically ventilated critically ill adults. Crit Care. 2014; 18 (6): 658. doi: 0.1186/s13054-014-0658-y.

10. Zang K, Chen B, Wang M, Chen D, Hui L, Guo S et al. The effect of early mobilization in critically ill patients: a meta-analysis. Nurs Crit Care. 2019; 1-8. https://doi.org/10.1111/nicc.12455.

11. Zhang L, Hu W, Cai Z, Liu J, Wu J, Deng Y et al. Early mobilization of critically ill patients in the intensive care unit: a systematic review and meta-analysis. PLoS ONE. 2019; 14 (10): e0223185. https://doi. org/10.1371/journal.pone.0223185.

12. Calixto AA, Mendoza NG, Martínez MA, Pérez OR, Deloya E, Martínez B. Movilización Temprana como prevención y tratamiento para la debilidad adquirida en la Unidad de Cuidados Intensivos en pacientes en ventilación mecánica. Experiencia en un hospital de segundo nivel. European Scientific Journal. 2018; 14 (21): ISSN: 1857-7881. doi: 10.19044/esj.2018.v14n21p19.

13. Namendys-Silva SA. Less is more, but are we doing enough? Intensive Care Medicine. 2020; 46: 113-115. doi: 10.1007/s00134-019-05831-7.

14. Ñamendys-Silva SA. Cuidados de alto valor en medicina crítica. Med Crit. 2019; 33 (2): 91-97.

15. Mehta S, Spies C, Shehabi Y. Ten tips for ICU sedation. Intensive Care Med. 2018; 44 (7): 1141-1143 doi: 10.1007/s00134-017-4992-9. 